MATHEMATICS OF COMPUTATION

Volume 78, Number 265, January 2009, Pages 241-253

S 0025-5718(08)02135-2

Article electronically published on May 16, 2008

\title{
ASYMPTOTIC EXPANSIONS OF GAUSS-LEGENDRE QUADRATURE RULES FOR INTEGRALS WITH ENDPOINT SINGULARITIES
}

\author{
AVRAM SIDI
}

This paper is dedicated to the memory of Professor Philip Rabinowitz

Abstract. Let $I[f]=\int_{-1}^{1} f(x) d x$, where $f \in C^{\infty}(-1,1)$, and let $G_{n}[f]=$ $\sum_{i=1}^{n} w_{n i} f\left(x_{n i}\right)$ be the $n$-point Gauss-Legendre quadrature approximation to $I[f]$. In this paper, we derive an asymptotic expansion as $n \rightarrow \infty$ for the error $E_{n}[f]=I[f]-G_{n}[f]$ when $f(x)$ has general algebraic-logarithmic singularities at one or both endpoints. We assume that $f(x)$ has asymptotic expansions of the forms

$$
\begin{aligned}
& f(x) \sim \sum_{s=0}^{\infty} U_{s}(\log (1-x))(1-x)^{\alpha_{s}} \quad \text { as } x \rightarrow 1-, \\
& f(x) \sim \sum_{s=0}^{\infty} V_{s}(\log (1+x))(1+x)^{\beta_{s}} \quad \text { as } x \rightarrow-1+,
\end{aligned}
$$

where $U_{s}(y)$ and $V_{s}(y)$ are some polynomials in $y$. Here, $\alpha_{s}$ and $\beta_{s}$ are, in general, complex and $\Re \alpha_{s}, \Re \beta_{s}>-1$. An important special case is that in which $U_{s}(y)$ and $V_{s}(y)$ are constant polynomials; for this case, the asymptotic expansion of $E_{n}[f]$ assumes the form

$$
E_{n}[f] \sim \sum_{\substack{s=0 \\ \alpha_{s} \notin \mathbb{Z}^{+}}}^{\infty} \sum_{i=1}^{\infty} a_{s i} h^{\alpha_{s}+i}+\sum_{\substack{s=0 \\ \beta_{s} \notin \mathbb{Z}^{+}}}^{\infty} \sum_{i=1}^{\infty} b_{s i} h^{\beta_{s}+i} \quad \text { as } n \rightarrow \infty,
$$

where $h=(n+1 / 2)^{-2}, \mathbb{Z}^{+}=\{0,1,2, \ldots\}$, and $a_{s i}$ and $b_{s i}$ are constants independent of $n$.

\section{INTRODUCTION}

Consider the problem of approximating finite-range integrals of the form

$$
I[f]=\int_{-1}^{1} f(x) d x
$$

by the $n$-point Gauss-Legendre quadrature rule

$$
G_{n}[f]=\sum_{i=1}^{n} w_{n i} f\left(x_{n i}\right)
$$

Received by the editor September 24, 2007 and, in revised form, January 10, 2008

2000 Mathematics Subject Classification. Primary 40A25, 41A55, 41A60, 65D30.

Key words and phrases. Gauss-Legendre quadrature, singular integrals, endpoint singularities, asymptotic expansions, Euler-Maclaurin expansions.

This research was supported in part by the United States-Israel Binational Science Foundation grant no. 2004353. 
where $x_{n i}$ are the abscissas [the zeros of $P_{n}(x)$, the $n$th Legendre polynomial] and $w_{n i}$ are the corresponding weights. Let

$$
E_{n}[f]=I[f]-G_{n}[f]
$$

denote the error in this approximation.

When $f \in C^{\infty}[-1,1]$, the error $E_{n}[f]$ tends to zero as $n \rightarrow \infty$ faster than all negative powers of $n$, that is, $E_{n}[f]=o\left(n^{-\mu}\right)$ as $n \rightarrow \infty$ for every $\mu>0$. In particular, when $f(z)$ is analytic in an open set of the $z$-plane that contains the interval $[-1,1]$ in its interior, it holds that $E_{n}[f]=O\left(e^{-\sigma n}\right)$ as $n \rightarrow \infty$ for some $\sigma>0$. See Davis and Rabinowitz [2, p. 312].

When $f(x)$ has integrable singularities in $(-1,1)$ and/or at one or both endpoints $x= \pm 1, E_{n}[f]$ tends to zero slowly, its rate of decay depending on the strength of the singularities. For example, when $f(x)=(1-x)^{\alpha} g(x)$, with $\Re \alpha>-1$ but $\alpha \neq 0,1, \ldots$, and $g \in C^{\infty}[-1,1]$, it is known that $E_{n}[f]=O\left(n^{-2 \alpha-2}\right)$ as $n \rightarrow \infty$. See [2, p. 313].

A much refined version of this result was given by Verlinden [11, Theorem 1]. For future reference, we reproduce Verlinden's result next:

Theorem 1.1. Let $f(x)=(1-x)^{\alpha} g(x)$, with $\Re \alpha>-1$ but $\alpha \neq 0,1, \ldots$, and $g(z)$ analytic in an open set that contains the interval $[-1,1]$ in its interior. Then, with $h=(n+1 / 2)^{-2}, E_{n}[f]$ has the asymptotic expansion

$$
E_{n}[f] \sim \sum_{k=1}^{\infty} a_{k} h^{\alpha+k} \quad \text { as } n \rightarrow \infty .
$$

Here, $a_{k}$ are some constants independent of $n$.

The proof of Verlinden's theorem is quite difficult and makes use of an important asymptotic result of Elliott [3] concerning the Jacobi polynomials and their corresponding functions of the second kind.

Interestingly, the asymptotic expansion in Theorem 1.1 resembles, in its form, the generalized Euler-Maclaurin expansion of Navot 4 for the trapezoidal rule approximation to the integral $\int_{-1}^{1}(1-x)^{\alpha} g(x) d x$. Thus, it could be viewed as an analogue of this Euler-Maclaurin expansion in the context of Gauss-Legendre quadrature. Verlinden 11 has also applied the Richardson extrapolation in conjunction with this expansion for approximating $\int_{-1}^{1}(1-x)^{\alpha} g(x) d x$ with high accuracy. For Euler-Maclaurin expansions and the Richardson extrapolation, see Atkinson [1], Ralston and Rabinowitz [7, Stoer and Bulirsch [10], and Sidi 8], for example.

In [11, Verlinden also gives an asymptotic expansion for the case in which $f(x)$ has an algebraic-logarithmic endpoint singularity. He considers specifically $f(x)=$ $\log (1-x)(1-x)^{\alpha} g(x)$, and shows that the asymptotic expansion of $E_{n}[f]$ in this case is obtained by differentiating that of Theorem 1.1 with respect to $\alpha$ term by term.

In this work, we consider functions $f(x)$ that have arbitrary algebraic-logarithmic endpoint singularities at one or both endpoints \pm 1 . The class of functions we consider is more general than that considered in [11, and contains the latter as a subclass. We derive asymptotic expansions of $E_{n}[f]$ as $n \rightarrow \infty$ for these functions. In the next section, we state our main results on these asymptotic expansions when $f \in C^{\infty}(-1,1)$ and mention some important special cases. In Section 3, we present the proofs of these results. In Section 4, we extend the results of Section 2 to the case in which $f(x)$ is only in $C^{r}(-1,1)$ for some nonnegative integer $r$. 


\section{Statement of MAIN Results}

Throughout this section, we assume that the function $f(x)$ in (1.1)-(1.3) has the following properties:

1. $f \in C^{\infty}(-1,1)$ and has the asymptotic expansions

$$
\begin{aligned}
& f(x) \sim \sum_{s=0}^{\infty} U_{s}(\log (1-x))(1-x)^{\alpha_{s}} \quad \text { as } x \rightarrow 1- \\
& f(x) \sim \sum_{s=0}^{\infty} V_{s}(\log (1+x))(1+x)^{\beta_{s}} \quad \text { as } x \rightarrow-1+
\end{aligned}
$$

where $U_{s}(y)$ and $V_{s}(y)$ are some polynomials in $y$, and $\alpha_{s}$ and $\beta_{s}$ are, in general, complex and satisfy

$$
\begin{aligned}
-1<\Re \alpha_{0} \leq \Re \alpha_{1} \leq \Re \alpha_{2} \leq \cdots ; & \lim _{s \rightarrow \infty} \Re \alpha_{s}=+\infty, \\
-1<\Re \beta_{0} \leq \Re \beta_{1} \leq \Re \beta_{2} \leq \cdots ; & \lim _{s \rightarrow \infty} \Re \beta_{s}=+\infty .
\end{aligned}
$$

Here, $\Re z$ stands for the real part of $z$.

2. If we let $u_{s}=\operatorname{deg}\left(U_{s}\right)$ and $v_{s}=\operatorname{deg}\left(V_{s}\right)$, then the $\alpha_{s}$ and $\beta_{s}$ are ordered such that

$$
u_{s} \geq u_{s+1} \quad \text { if } \quad \Re \alpha_{s+1}=\Re \alpha_{s} ; \quad v_{s} \geq v_{s+1} \quad \text { if } \quad \Re \beta_{s+1}=\Re \beta_{s} .
$$

3. By (2.1), we mean that, for each $r=1,2, \ldots$,

$$
\begin{gathered}
\begin{array}{c}
f(x)-\sum_{s=0}^{r-1} U_{s}(\log (1-x))(1-x)^{\alpha_{s}} \\
=O\left(U_{r}(\log (1-x))(1-x)^{\alpha_{r}}\right) \quad \text { as } x \rightarrow 1- \\
f(x)-\sum_{s=0}^{r-1} V_{s}(\log (1+x))(1+x)^{\beta_{s}} \\
=O\left(V_{r}(\log (1+x))(1+x)^{\beta_{r}}\right) \quad \text { as } x \rightarrow-1+.
\end{array}
\end{gathered}
$$

4. For each $k=1,2, \ldots$, the $k$ th derivative of $f(x)$ also has asymptotic expansions as $x \rightarrow \pm 1$ that are obtained by differentiating those in (2.1) term by term.

The following are consequences of (2.2) and (2.3):

(i) There are only a finite number of $\alpha_{s}$ that have the same real parts. Similarly, there are only a finite number of $\beta_{s}$ that have the same real parts. Consequently, $\Re \alpha_{s}<\Re \alpha_{s+1}$ and $\Re \beta_{s^{\prime}}<\Re \beta_{s^{\prime}+1}$ for infinitely many values of the indices $s$ and $s^{\prime}$.

(ii) The sequences $\left\{U_{s}(\log (1-x))(1-x)^{\alpha_{s}}\right\}_{s=0}^{\infty}$ and $\left\{V_{s}(\log (1+x))(1+x)^{\beta_{s}}\right\}_{s=0}^{\infty}$ are asymptotic scales. For a discussion of asymptotic scales, see Olver [5, p. 25], for example. Thus, also by (2.4), the expansions in (2.1) are genuine asymptotic expansions.

A key result that we will use to state and prove our main theorems is essentially given in [11, Section 6]; it is also a corollary of Theorem 1.1 corresponding to the case $g(x) \equiv 1$ there. We state it below as Theorem 2.1. To that effect, let us define

$$
f_{\omega}^{ \pm}(x)=(1 \pm x)^{\omega}
$$


Then

$$
I\left[f_{\omega}^{+}\right]=I\left[f_{\omega}^{-}\right]=\frac{2^{\omega+1}}{\omega+1}, \quad \Re \omega>-1 .
$$

By the symmetry of the Gauss-Legendre quadrature formulas, namely, by the fact that $x_{n, n-i+1}=-x_{n i}$ and $w_{n, n-i+1}=w_{n i}$ for all $i$ in (1.2), we also have

$$
G_{n}\left[f_{\omega}^{+}\right]=G_{n}\left[f_{\omega}^{-}\right]
$$

so that

$$
E_{n}\left[f_{\omega}^{+}\right]=E_{n}\left[f_{\omega}^{-}\right] .
$$

Theorem 2.1. Let $f_{\omega}^{ \pm}(x)$ be as in (2.5), with $\Re \omega>-1$ but $\omega \notin \mathbb{Z}^{+}$, where $\mathbb{Z}^{+}=$ $\{0,1,2, \ldots\}$. Then, with $h=(n+1 / 2)^{-2}, E_{n}\left[f_{\omega}^{ \pm}\right]$has the asymptotic expansion

$$
E_{n}\left[f_{\omega}^{ \pm}\right] \sim \sum_{k=1}^{\infty} c_{k}(\omega) h^{\omega+k} \quad \text { as } n \rightarrow \infty,
$$

that is valid uniformly in every strip $-1<d_{1} \leq \Re \omega \leq d_{2}<\infty$ of the $\omega$-plane. The $c_{k}(\omega)$ are analytic functions of $\omega$ for $\Re \omega>-1$ (same functions for $f_{\omega}^{+}$and for $f_{\omega}^{-}$). When $\omega \in \mathbb{Z}^{+}$, for each $k=0,1, \ldots$, it holds that $c_{k}(\omega)=0$; in this case, we also have $E_{n}\left[f_{\omega}^{ \pm}\right]=0$ for all $n \geq(\omega+1) / 2$.

We now state the main results of this work. We start with the following special case of pure algebraic (nonlogarithmic) endpoint singularities that is important and of interest in itself:

Theorem 2.2. Let $f(x)$ be exactly as described in the first paragraph of this section with the same notation, $U_{s}(y)=A_{s} \neq 0$ and $V_{s}(y)=B_{s} \neq 0$ being constant polynomials for all s. Then, with $h=(n+1 / 2)^{-2}$ and $\mathbb{Z}^{+}=\{0,1,2, \ldots\}$, it holds that

$$
E_{n}[f] \sim \sum_{\substack{s=0 \\ \alpha_{s} \notin \mathbb{Z}^{+}}}^{\infty} A_{s} \sum_{k=1}^{\infty} c_{k}\left(\alpha_{s}\right) h^{\alpha_{s}+k}+\sum_{\substack{s=0 \\ \beta_{s} \notin \mathbb{Z}^{+}}}^{\infty} B_{s} \sum_{k=1}^{\infty} c_{k}\left(\beta_{s}\right) h^{\beta_{s}+k} \quad \text { as } n \rightarrow \infty .
$$

Here, $c_{k}(\omega)$ are precisely as given in Theorem 2.1 ,

Remarks.

1. By (2.2), the sequences $\left\{h^{\alpha_{s}+k}\right\}_{s=0}^{\infty}$ and $\left\{h^{\beta_{s}+k}\right\}_{s=0}^{\infty}$ are asymptotic scales as $n \rightarrow \infty$, and the expansion in (2.10) is a genuine asymptotic expansion when its terms are reordered according to their size.

2. Note that, when $U_{s}(y)$ and $V_{s}(y)$ are constants, the nonnegative integer powers $(1-x)^{s}$ and $(1+x)^{s}$, if present in the asymptotic expansions of (2.1), do not contribute to the expansion of $E_{n}[f]$ as $n \rightarrow \infty$.

3. In case $\alpha_{s}, \beta_{s}$ are all nonnegative integers in Theorem 2.2. of course, $f \in$ $C^{\infty}[-1,1]$, and the asymptotic expansion in (2.10) is empty (zero). This does not necessarily mean that $E_{n}[f]=0$, however. It only means that $E_{n}[f]$ tends to zero as $n \rightarrow \infty$ faster than all negative powers of $n$, which is consistent with the known result we mentioned in Section 1 Of course, when $f(x)$ is a polynomial, $E_{n}[f]=0$ for all large $n$.

4. If $\alpha_{s}=\alpha+s$ and $\beta_{s}=s$ for all $s=0,1, \ldots$, in Theorem 2.2, then $f(x)$ is of the form $f(x)=(1-x)^{\alpha} g(x)$ with $g \in C^{\infty}[-1,1]$, and $A_{s}=(-1)^{s} g^{(s)}(1) / s !$, 
$s=0,1, \ldots$. In this case, the second double sum in (2.10) disappears and the first double sum can be rearranged so that

$$
E_{n}[f] \sim \sum_{k=1}^{\infty} a_{k} h^{\alpha+k} \quad \text { as } n \rightarrow \infty
$$

where $a_{k}$ are functions of $\alpha$ given by

$$
a_{k}=\left[\sum_{s=0}^{k-1} A_{s} c_{k-s}(\alpha+k)\right], \quad k=1,2, \ldots,
$$

and are analytic in every strip $-1<d_{1} \leq \Re \alpha \leq d_{2}<\infty$ of the $\alpha$-plane. Thus, Theorem 2.2 reduces to the result of Verlinden given in Theorem 1.1. however, it is more general since the function $g(z)$ now is not assumed to be analytic in an open set in the $z$-plane containing the interval $[-1,1]$ but is assumed to be in $C^{\infty}[-1,1]$ only.

5. If $\alpha_{s}=\alpha+s$ and $\beta_{s}=\beta+s$ for all $s=0,1, \ldots$, in Theorem 2.2, then $f(x)$ is of the form $f(x)=(1-x)^{\alpha}(1+x)^{\beta} g(x)$ with $g \in C^{\infty}[-1,1]$, and $A_{s}$ and $B_{s}$ are given by

$$
\begin{aligned}
& A_{s}=\left.\frac{(-1)^{s}}{s !} \frac{d^{s}}{d x^{s}}\left[(1+x)^{\beta} g(x)\right]\right|_{x=1}=(-1)^{s} \sum_{i=0}^{s}\left(\begin{array}{c}
\beta \\
i
\end{array}\right) \frac{g^{(s-i)}(1)}{(s-i) !} 2^{\beta-i} \\
& B_{s}=\left.\frac{1}{s !} \frac{d^{s}}{d x^{s}}\left[(1-x)^{\alpha} g(x)\right]\right|_{x=-1}=\sum_{i=0}^{s}(-1)^{i}\left(\begin{array}{c}
\alpha \\
i
\end{array}\right) \frac{g^{(s-i)}(-1)}{(s-i) !} 2^{\alpha-i}
\end{aligned}
$$

Note that $A_{s}$ are entire functions of $\beta$ only, while $B_{s}$ are entire functions of $\alpha$ only. In this case, by rearranging both of the double sums in (2.10), we have the following generalization of Theorem 1.1 for algebraic singularities at both endpoints:

$$
E_{n}[f] \sim \sum_{k=1}^{\infty} a_{k} h^{\alpha+k}+\sum_{k=1}^{\infty} b_{k} h^{\beta+k} \quad \text { as } n \rightarrow \infty .
$$

Here, $a_{k}$ and $b_{k}$ are functions of both $\alpha$ and $\beta$ given by

$$
a_{k}=\sum_{s=0}^{k-1} A_{s} c_{k-s}(\alpha+k), \quad b_{k}=\sum_{s=0}^{k-1} B_{s} c_{k-s}(\beta+k), \quad k=1,2, \ldots,
$$

and are analytic when $\alpha$ and $\beta$ are such that $-1<d_{1} \leq \Re \alpha \leq d_{2}<\infty$ and $-1<d_{1}^{\prime} \leq \Re \beta \leq d_{2}^{\prime}<\infty$, respectively.

The next theorem deals with the general case, in which algebraic-logarithmic singularities may occur at the endpoints.

Theorem 2.3. Let $f(x)$ be exactly as described in the first paragraph of this section with the same notation, and let $U_{s}(y)=\sum_{i=0}^{u_{s}} \sigma_{s i} y^{i}$ and $V_{s}(y)=\sum_{i=0}^{v_{s}} \tau_{s i} y^{i}$. Denote $\frac{d}{d \omega}$ by $D_{\omega}$. For an arbitrary polynomial $W(y)=\sum_{i=0}^{k} \epsilon_{i} y^{i}$ and an arbitrary function $g$ that depends on $\omega$, define also

$$
W\left(D_{\omega}\right) g:=\sum_{i=0}^{k} \epsilon_{i}\left[D_{\omega}^{i} g\right]=\sum_{i=0}^{k} \epsilon_{i} \frac{d^{i} g}{d \omega^{i}} .
$$


Then, with $h=(n+1 / 2)^{-2}$, it holds that

$$
\begin{aligned}
E_{n}[f] \sim & \sum_{s=0}^{\infty} \sum_{k=1}^{\infty} U_{s}\left(D_{\alpha_{s}}\right)\left[c_{k}\left(\alpha_{s}\right) h^{\alpha_{s}+k}\right] \\
& +\sum_{s=0}^{\infty} \sum_{k=1}^{\infty} V_{s}\left(D_{\beta_{s}}\right)\left[c_{k}\left(\beta_{s}\right) h^{\beta_{s}+k}\right] \quad \text { as } n \rightarrow \infty .
\end{aligned}
$$

Here, $c_{k}(\omega)$ are precisely as given in Theorem 2.1 .

\section{Remarks.}

1. To see the explicit form of the expansion in Theorem 2.3. we also need

$$
D_{\omega}^{i}\left[c_{k}(\omega) h^{\omega+k}\right]=h^{\omega+k} \sum_{j=0}^{i}\left(\begin{array}{l}
i \\
j
\end{array}\right) c_{k}^{(i-j)}(\omega)(\log h)^{j},
$$

where $c_{k}^{(r)}(\omega)$ stands for the $r$ th derivative of $c_{k}(\omega)$. Using this, it can be seen, for example, that

$$
U_{s}\left(D_{\alpha_{s}}\right)\left[c_{k}\left(\alpha_{s}\right) h^{\alpha_{s}+k}\right]=h^{\alpha_{s}+k} \sum_{j=0}^{u_{s}} e_{s j}(\log h)^{j},
$$

where

$$
e_{s j}=\sum_{i=j}^{u_{s}}\left(\begin{array}{l}
i \\
j
\end{array}\right) \sigma_{s i} c_{k}^{(i-j)}\left(\alpha_{s}\right), \quad j=0,1, \ldots, u_{s} .
$$

Note that $e_{s u_{s}}=\sigma_{s u_{s}} c_{k}\left(\alpha_{s}\right)$. By Theorem 2.1 this implies that $e_{s u_{s}}=0$ when $\alpha_{s} \in \mathbb{Z}^{+}$.

Thus, (2.16) assumes the following explicit form:

$$
E_{n}[f] \sim \sum_{s=0}^{\infty} \sum_{k=1}^{\infty} \widehat{U}_{s k}(\log h) h^{\alpha_{s}+k}+\sum_{s=0}^{\infty} \sum_{k=1}^{\infty} \widehat{V}_{s k}(\log h) h^{\beta_{s}+k} \quad \text { as } n \rightarrow \infty,
$$

where $\widehat{U}_{s k}(y)$ and $\widehat{V}_{s k}(y)$ are polynomials in $y$ with $\operatorname{deg}\left(\widehat{U}_{s k}\right) \leq u_{s}$ and $\operatorname{deg}\left(V_{s}\right) \leq v_{s}$. If $\alpha_{s} \in \mathbb{Z}^{+}$, then $\operatorname{deg}\left(\widehat{U}_{s k}\right) \leq u_{s}-1$; otherwise, $\operatorname{deg}\left(\widehat{U}_{s k}\right)=$ $u_{s}$. Similarly, if $\beta_{s} \in \mathbb{Z}^{+}$, then $\operatorname{deg}\left(\widehat{V}_{s k}\right) \leq v_{s}-1$; otherwise, $\operatorname{deg}\left(\widehat{V}_{s k}\right)=v_{s}$.

2. Invoking now (2.2) and (2.3), we conclude that the sequences

$$
\left\{U_{s}\left(D_{\alpha_{s}}\right)\left[c_{k}\left(\alpha_{s}\right) h^{\alpha_{s}+k}\right]\right\}_{s=0}^{\infty} \text { and }\left\{V_{s}\left(D_{\beta_{s}}\right)\left[c_{k}\left(\beta_{s}\right) h^{\beta_{s}+k}\right]\right\}_{s=0}^{\infty}
$$

are asymptotic scales as $n \rightarrow \infty$, and that the expansion in (2.16) is a genuine asymptotic expansion.

3. When $\alpha_{s}=\alpha+s$ and $\beta_{s}=\beta+s$, for all $s=0,1, \ldots$, and $u_{0}=u_{1}=\cdots=p$ and $v_{0}=v_{1}=\cdots=q$, we can rearrange the double sums in (2.17), and obtain

$$
E_{n}[f] \sim \sum_{k=1}^{\infty} \check{U}_{k}(\log h) h^{\alpha+k}+\sum_{k=1}^{\infty} \check{V}_{k}(\log h) h^{\beta+k} \quad \text { as } n \rightarrow \infty,
$$

where $\check{U}_{k}(y)=\sum_{s=0}^{k-1} \widehat{U}_{s, k-s}(y)$ and $\check{V}_{k}(y)=\sum_{s=0}^{k-1} \widehat{V}_{s, k-s}(y)$ are polynomials in $y$ of degree at most $p$ and $q$, respectively. If $\alpha \in \mathbb{Z}^{+}$, then $\operatorname{deg}\left(\check{U}_{k}\right) \leq p-1$. Similarly, if $\beta \in \mathbb{Z}^{+}$, then $\operatorname{deg}\left(\check{V}_{k}\right) \leq q-1$. 
4. The case in the preceding remark arises, for example, when

$$
f(x)=(1-x)^{\alpha}(1+x)^{\beta}[\log (1-x)]^{p}[\log (1+x)]^{q} g(x)
$$

with $g \in C^{\infty}[-1,1]$. In this case, the asymptotic expansion of $E_{n}[f]$ can be obtained by differentiating the asymptotic expansion of $E_{n}[\widetilde{f}]$, where $\widetilde{f}(x)=(1-x)^{\alpha}(1+x)^{\beta} g(x), p$ times with respect to $\alpha$ and $q$ times with respect to $\beta$. Note that $\widetilde{f}(x)$ here is precisely the function $f(x)$ given in Remark 5 following Theorem 2.2 , and the asymptotic expansion of $E_{n}[\widetilde{f}]$ is as given in (2.14) and (2.15). Recall that the $a_{k}$ and $b_{k}$ there are analytic functions of both $\alpha$ and $\beta$. Thus, applying $\partial^{p+q} / \partial \alpha^{p} \partial \beta^{q}$ to (2.14), we obtain the expansion in (2.18).

A simpler special case is one in which $\beta=0$ and $q=0$. For this case, we have $f(x)=(1-x)^{\alpha}[\log (1-x)]^{p} g(x)$ with $p$ a positive integer and $g \in C^{\infty}[-1,1]$. The asymptotic expansion of $E_{n}[f]$ is now of the form

$$
E_{n}[f] \sim \sum_{k=1}^{\infty} \check{U}_{k}(\log h) h^{\alpha+k} \quad \text { as } n \rightarrow \infty,
$$

where $\check{U}_{k}(y)$, as before, are polynomials in $y$ of degree at most $p$, and this can be obtained by differentiating the asymptotic expansion of $E_{n}[\widetilde{f}]$, where $\widetilde{f}(x)=(1-x)^{\alpha} g(x), p$ times with respect to $\alpha$. The asymptotic expansion of $E_{n}[\tilde{f}]$ is that given in (2.11). The case $p=1$ has been given in 11, Section 6].

Just as the expansion of $E_{n}[f]$ (for Gauss-Legendre quadrature) in Theorem 1.1 is an analogue of Navot's generalized Euler-Maclaurin expansion (for the trapezoidal rule), those in Theorems 2.2 and 2.3 (for Gauss-Legendre quadrature) are analogues of the author's [9] recent generalizations of the Euler-Maclaurin expansion (for the trapezoidal rule) under precisely the same conditions.

\section{Proofs of MAin Results}

3.1. Proof of Theorem 2.2, With $U_{s}(y)=A_{s}$ and $V_{s}(y)=B_{s}$, and an arbitrary positive integer $m$, let

$$
p(x)=\sum_{s=0}^{m-1} A_{s}(1-x)^{\alpha_{s}}+\sum_{s=0}^{m-1} B_{s}(1+x)^{\beta_{s}}=\sum_{s=0}^{m-1} A_{s} f_{\alpha_{s}}^{-}(x)+\sum_{s=0}^{m-1} B_{s} f_{\beta_{s}}^{+}(x) .
$$

Here, $f_{\omega}^{ \pm}(x)$ are as defined in (2.5). Then,

$$
f(x)=p(x)+\phi(x) ; \quad \phi(x):=f(x)-p(x) .
$$

Thus,

$$
E_{n}[f]=E_{n}[p]+E_{n}[\phi] .
$$

By Theorem 2.1.

$$
\begin{aligned}
E_{n}[p] & =\sum_{s=0}^{m-1} A_{s} E_{n}\left[f_{\alpha_{s}}^{-}\right]+\sum_{s=0}^{m-1} B_{s} E_{n}\left[f_{\beta_{s}}^{+}\right] \\
& \sim \sum_{s=0}^{m-1} A_{s} \sum_{k=1}^{\infty} c_{k}\left(\alpha_{s}\right) h^{\alpha_{s}+k}+\sum_{s=0}^{m-1} B_{s} \sum_{k=1}^{\infty} c_{k}\left(\beta_{s}\right) h^{\beta_{s}+k} \quad \text { as } n \rightarrow \infty .
\end{aligned}
$$


We now have to analyze $E_{n}[\phi]$. For this, we need to know the differentiability properties of $\phi(x)$ on $[-1,1]$. First, $\phi \in C^{\infty}(-1,1)$. At $x= \pm 1, \phi(x)$ has the asymptotic expansions

$$
\begin{aligned}
& \phi(x) \sim w_{m}^{+}(x)+\sum_{s=m}^{\infty} A_{s}(1-x)^{\alpha_{s}} \quad \text { as } x \rightarrow 1-; \quad w_{m}^{+}(x)=-\sum_{s=0}^{m-1} B_{s}(1+x)^{\beta_{s}}, \\
& \phi(x) \sim w_{m}^{-}(x)+\sum_{s=m}^{\infty} B_{s}(1+x)^{\beta_{s}} \quad \text { as } x \rightarrow-1+; \quad w_{m}^{-}(x)=-\sum_{s=0}^{m-1} A_{s}(1-x)^{\alpha_{s}} .
\end{aligned}
$$

Note that $w_{m}^{+}(x)$ is infinitely differentiable at $x=1$ while $w_{m}^{-}(x)$ is infinitely differentiable at $x=-1$. Thus, what determines the differentiability properties on $[-1,1]$ of $\phi(x)$ are the infinite sums in (3.5). By the fourth of the properties of $f(x)$ mentioned in the beginning of Section 2, the asymptotic expansions of $\phi(x)$ in (3.5) can be differentiated termwise as many times as we wish. Then, for every positive integer $j$, it holds that

$$
\begin{aligned}
\frac{d^{j}}{d x^{j}} \phi(x) \sim & \frac{d^{j}}{d x^{j}} w_{m}^{+}(x) \\
& +\sum_{s=m}^{\infty} A_{s} \alpha_{s}\left(\alpha_{s}-1\right) \cdots\left(\alpha_{s}-j+1\right)(1-x)^{\alpha_{s}-j} \quad \text { as } x \rightarrow 1-, \\
\frac{d^{j}}{d x^{j}} \phi(x) \sim & \frac{d^{j}}{d x^{j}} w_{m}^{-}(x) \\
& +\sum_{s=m}^{\infty} B_{s} \beta_{s}\left(\beta_{s}-1\right) \cdots\left(\beta_{s}-j+1\right)(1+x)^{\beta_{s}-j} \quad \text { as } x \rightarrow-1+.
\end{aligned}
$$

Clearly,

$$
\begin{aligned}
& \lim _{x \rightarrow 1-} \frac{d^{j}}{d x^{j}} \phi(x)=-\left.\frac{d^{j} w_{m}^{+}}{d x^{j}}\right|_{x=1}, \quad j=0,1, \ldots,\left\lceil\Re \alpha_{m}-1\right\rceil, \\
& \lim _{x \rightarrow-1+} \frac{d^{j}}{d x^{j}} \phi(x)=-\left.\frac{d^{j} w_{m}^{-}}{d x^{j}}\right|_{x=-1}, \quad j=0,1, \ldots,\left\lceil\Re \beta_{m}-1\right\rceil,
\end{aligned}
$$

which also means that $\phi(x)$ has $\left\lceil\Re \alpha_{m}-1\right\rceil$ continuous derivatives at $x=1$ and $\left\lceil\Re \beta_{m}-1\right\rceil$ continuous derivatives at $x=-1$, in addition to being in $C^{\infty}(-1,1)$. Consequently, $\phi \in C^{\kappa_{m}}[-1,1]$, where $\kappa_{m}=\min \left\{\left\lceil\Re \alpha_{m}-1\right\rceil,\left\lceil\Re \beta_{m}-1\right\rceil\right\}$.

Next, it is known that

$$
\left|E_{n}[\phi]\right| \leq 4 \min _{q \in \Pi_{2 n-1}}\|\phi-q\|,
$$

where $\Pi_{k}$ is the set of all polynomials of degree at most $k$ and

$$
\|F\|=\max _{x \in[-1,1]}|F(x)|
$$

and that

$$
\min _{q \in \Pi_{N}}\|F-q\|=O\left(N^{-k}\right) \quad \text { as } N \rightarrow \infty, \quad \text { when } F \in C^{k}[-1,1],
$$

by one of Jackson's theorems. For (3.8), see [2, Section 4.8, pp. 332-333], and for Jackson's theorem leading to (3.9), see Powell [6, Section 16.3, pp. 194-198], for 
example. Thus,

$$
\min _{q \in \Pi_{2 n-1}}\|\phi-q\|=O\left(n^{-\kappa_{m}}\right)=O\left(h^{\kappa_{m} / 2}\right) \quad \text { as } n \rightarrow \infty .
$$

From (3.8) and (3.10), it therefore follows that

$$
E_{n}[\phi]=O\left(h^{\kappa_{m} / 2}\right) \quad \text { as } n \rightarrow \infty .
$$

Combining (3.4) and (3.11) in (3.3), and considering only those terms with $\Re \alpha_{s}+$ $k<\Re \alpha_{m}$ and $\Re \beta_{s}+k<\Re \beta_{m}$ in, respectively, the first and second double summations in (3.4), we have

$$
\begin{aligned}
E_{n}[f]= & \sum_{\substack{0 \leq s \leq m-1 \\
1 \leq k<\Re\left(\alpha_{m}-\alpha_{s}\right)}} A_{s} c_{k}\left(\alpha_{s}\right) h^{\alpha_{s}+k}+O\left(h^{\alpha_{m}}\right) \\
& +\sum_{\substack{0 \leq s \leq m-1 \\
1 \leq k<\Re\left(\beta_{m}-\beta_{s}\right)}}^{m-1} B_{s} c_{k}\left(\beta_{s}\right) h^{\beta_{s}+k}+O\left(h^{\beta_{m}}\right) \\
& +O\left(h^{\kappa_{m} / 2}\right) \text { as } n \rightarrow \infty .
\end{aligned}
$$

Now, $\lim _{m \rightarrow \infty} \kappa_{m}=\infty$ and $\lim _{m \rightarrow \infty} \Re \alpha_{m}=\infty$ and $\lim _{m \rightarrow \infty} \Re \beta_{m}=\infty$ simultaneously, by (2.2). From this and from (3.12), we conclude that $E_{n}[f]$ has the true asymptotic expansion

$$
E_{n}[f] \sim \sum_{s=0}^{\infty} A_{s} \sum_{k=1}^{\infty} c_{k}\left(\alpha_{s}\right) h^{\alpha_{s}+k}+\sum_{s=0}^{\infty} B_{s} \sum_{k=1}^{\infty} c_{k}\left(\beta_{s}\right) h^{\beta_{s}+k} \quad \text { as } n \rightarrow \infty .
$$

Finally, the result in (2.10) follows by invoking the fact that $c_{k}(\omega)=0$ when $\omega \in \mathbb{Z}^{+}$.

3.2. Proof of Theorem 2.3. We first observe that, with $f_{\omega}^{ \pm}(x)$ as defined in (2.5),

$$
f_{\omega, i}^{ \pm}(x):=[\log (1 \pm x)]^{i}(1 \pm x)^{\omega}=\frac{d^{i}}{d \omega^{i}} f_{\omega}^{ \pm}(x) .
$$

Consequently, we also have

$$
I\left[f_{\omega, i}^{ \pm}\right]=\frac{d^{i}}{d \omega^{i}} I\left[f_{\omega}^{ \pm}\right]=\frac{d^{i}}{d \omega^{i}} \frac{2^{\omega+1}}{\omega+1}
$$

and

$$
G_{n}\left[f_{\omega, i}^{ \pm}\right]=\frac{d^{i}}{d \omega^{i}} G_{n}\left[f_{\omega}^{ \pm}\right]
$$

and hence

$$
E_{n}\left[f_{\omega, i}^{ \pm}\right]=\frac{d^{i}}{d \omega^{i}} E_{n}\left[f_{\omega}^{ \pm}\right]
$$

The following theorem, which we employ in our proof, essentially follows from [11, Section 6].

Theorem 3.1. Let $\Re \omega>-1$. Then, with $h=(n+1 / 2)^{-2}$, for each $i=1,2, \ldots$, $E_{n}\left[f_{\omega, i}^{ \pm}\right]$has the asymptotic expansion

$$
E_{n}\left[f_{\omega, i}^{ \pm}\right] \sim \sum_{k=1}^{\infty} \frac{d^{i}}{d \omega^{i}}\left[c_{k}(\omega) h^{\omega+k}\right] \quad \text { as } n \rightarrow \infty,
$$

that is valid uniformly in every strip $-1<d_{1} \leq \Re \omega \leq d_{2}<\infty$ of the $\omega$-plane. 
Remark. In other words, the asymptotic expansion of $E_{n}\left[f_{\omega, i}^{ \pm}\right]$is obtained by differentiating that of $E_{n}\left[f_{\omega}^{ \pm}\right] i$ times term by term. Note, however, that even though $c_{k}(\omega)$ vanish when $\omega \in \mathbb{Z}^{+}, c_{k}^{(i)}(\omega)$ do not have to.

For an arbitrary positive integer $m$, let

$$
\begin{aligned}
p(x) & =\sum_{s=0}^{m-1} U_{s}(\log (1-x))(1-x)^{\alpha_{s}}+\sum_{s=0}^{m-1} V_{s}(\log (1+x))(1+x)^{\beta_{s}} \\
& =\sum_{s=0}^{m-1} \sum_{i=0}^{u_{s}} \sigma_{s i} f_{\alpha_{s}, i}^{-}(x)+\sum_{s=0}^{m-1} \sum_{i=0}^{v_{s}} \tau_{s i} f_{\beta_{s}, i}^{+}(x),
\end{aligned}
$$

and write, as before,

$$
f(x)=p(x)+\phi(x) ; \quad \phi(x):=f(x)-p(x),
$$

and

$$
E_{n}[f]=E_{n}[p]+E_{n}[\phi],
$$

However, this time,

$$
E_{n}[p]=\sum_{s=0}^{m-1} \sum_{i=0}^{u_{s}} \sigma_{s i} E_{n}\left[f_{\alpha_{s}, i}^{-}\right]+\sum_{s=0}^{m-1} \sum_{i=0}^{v_{s}} \tau_{s i} E_{n}\left[f_{\beta_{s}, i}^{+}\right] .
$$

By Theorem 3.1, this gives

$$
\begin{aligned}
E_{n}[p] \sim & \sum_{s=0}^{m-1} \sum_{k=1}^{\infty} U_{s}\left(D_{\alpha_{s}}\right)\left[c_{k}\left(\alpha_{s}\right) h^{\alpha_{s}+k}\right] \\
& +\sum_{s=0}^{m-1} \sum_{k=1}^{\infty} V_{s}\left(D_{\beta_{s}}\right)\left[c_{k}\left(\beta_{s}\right) h^{\beta_{s}+k}\right] \quad \text { as } n \rightarrow \infty .
\end{aligned}
$$

To analyze $E_{n}[\phi]$, we again need to study the differentiability properties of $\phi(x)$ on $[-1,1]$. Clearly, $\phi \in C^{\infty}(-1,1)$. At $x= \pm 1, \phi(x)$ has the asymptotic expansions

$$
\begin{aligned}
& \phi(x) \sim w_{m}^{+}(x)+\sum_{s=m}^{\infty} \sum_{i=0}^{u_{s}} \sigma_{s i}[\log (1-x)]^{i}(1-x)^{\alpha_{s}} \quad \text { as } x \rightarrow 1-, \\
& \phi(x) \sim w_{m}^{-}(x)+\sum_{s=m}^{\infty} \sum_{i=0}^{v_{s}} \tau_{s i}[\log (1+x)]^{i}(1+x)^{\beta_{s}} \quad \text { as } x \rightarrow-1+,
\end{aligned}
$$

with

$$
\begin{aligned}
& w_{m}^{+}(x)=-\sum_{s=0}^{m-1} V_{s}(\log (1+x))(1+x)^{\beta_{s}}, \\
& w_{m}^{-}(x)=-\sum_{s=0}^{m-1} U_{s}(\log (1-x))(1-x)^{\alpha_{s}} .
\end{aligned}
$$

As was the case in the proof of Theorem 2.2, again $w_{m}^{+}(x)$ is infinitely differentiable at $x=1$ while $w_{m}^{-}(x)$ is infinitely differentiable at $x=-1$. By the fourth of the properties of $f(x)$ mentioned in the beginning of Section 2 the asymptotic 
expansions of $\phi(x)$ in (3.24) can be differentiated termwise as many times as we wish. Then, for every positive integer $j$, it holds that

$$
\begin{aligned}
& \frac{d^{j}}{d x^{j}} \phi(x) \sim \frac{d^{j}}{d x^{j}} w_{m}^{+}(x)+\sum_{s=m}^{\infty} \widetilde{U}_{s}(\log (1-x))(1-x)^{\alpha_{s}-j} \quad \text { as } x \rightarrow 1-, \\
& \frac{d^{j}}{d x^{j}} \phi(x) \sim \frac{d^{j}}{d x^{j}} w_{m}^{-}(x)+\sum_{s=m}^{\infty} \widetilde{V}_{s}(\log (1+x))(1+x)^{\beta_{s}-j} \quad \text { as } x \rightarrow-1+,
\end{aligned}
$$

where $\widetilde{U}_{s}(y)$ and $\widetilde{V}_{s}(y)$ are polynomials in $y$ of degree $u_{s}$ and $v_{s}$, respectively. It is easy to see that, in this case too, we have

$$
\begin{aligned}
& \lim _{x \rightarrow 1-} \frac{d^{j}}{d x^{j}} \phi(x)=-\left.\frac{d^{j} w_{m}^{+}}{d x^{j}}\right|_{x=1}, \quad j=0,1, \ldots,\left\lceil\Re \alpha_{m}-1\right\rceil, \\
& \lim _{x \rightarrow-1+} \frac{d^{j}}{d x^{j}} \phi(x)=-\left.\frac{d^{j} w_{m}^{-}}{d x^{j}}\right|_{x=-1}, \quad j=0,1, \ldots,\left\lceil\Re \beta_{m}-1\right\rceil,
\end{aligned}
$$

which also means that $\phi(x)$ has $\left\lceil\Re \alpha_{m}-1\right\rceil$ continuous derivatives at $x=1$ and $\left\lceil\Re \beta_{m}-1\right\rceil$ continuous derivatives at $x=-1$, in addition to being in $C^{\infty}(-1,1)$. Consequently, $\phi \in C^{\kappa_{m}}[-1,1]$, where $\kappa_{m}=\min \left\{\left\lceil\Re \alpha_{m}-1\right\rceil,\left\lceil\Re \beta_{m}-1\right\rceil\right\}$.

The proof of Theorem 2.3 can now be completed as that of Theorem 2.2. We leave the details to the reader.

\section{Extensions}

In the preceding sections, we assumed that the function $f(x)$ is infinitely differentiable on $(-1,1)$. However, the proofs of Theorems 2.2 and 2.3 suggest that these theorems can be extended to the case in which the function $f(x)$ is not necessarily in $C^{\infty}(-1,1)$.

Theorems 4.1 and 4.2 below are extensions of Theorems 2.2 and 2.3 , respectively, precisely to this case. In these theorems, we assume that $f(x)$ is exactly as in the first paragraph of Section 2, except that it ceases to be infinitely differentiable at a finite number of points in $(-1,1)$, and that it is in $C^{r}(-1,1)$ for some nonnegative integer $r$. Of course, $f(x)$ continues to be infinitely differentiable in the open intervals $(-1,-1+\eta)$ and $(1-\eta, 1)$, where $\eta$ is sufficiently small and, in addition, as $x \rightarrow \pm 1, f(x)$ has the asymptotic expansions given in (2.1), with (2.2)-(2.4). Below, we adopt the notation of Sections 2 and 3 .

Theorem 4.1. Let $f(x)$ be as in the second paragraph of this section with the same notation, $U_{s}(y)=A_{s} \neq 0$ and $V_{s}(y)=B_{s} \neq 0$ being constant polynomials for all $s$. Let $m_{-}$and $m_{+}$be the smallest integers for which

$$
r<\Re \alpha_{m_{-}} \text {and } r<\Re \beta_{m_{+}} .
$$

Then, with $h=(n+1 / 2)^{-2}$ and $\mathbb{Z}^{+}=\{0,1,2, \ldots\}$, it holds that

$$
\begin{aligned}
E_{n}[f]=\sum_{\substack{s=0 \\
\alpha_{s} \notin \mathbb{Z}^{+}}}^{m_{-}-1} \sum_{k=1}^{\left\lceil r / 2-\Re \alpha_{s}-1\right\rceil} A_{s} c_{k}\left(\alpha_{s}\right) h^{\alpha_{s}+k} & \\
& +\sum_{\substack{s=0 \\
\beta_{s} \notin \mathbb{Z}^{+}}}^{m_{+}-1} \sum_{k=1}^{\left\lceil r / 2-\Re \beta_{s}-1\right\rceil} B_{s} c_{k}\left(\beta_{s}\right) h^{\beta_{s}+k}+O\left(h^{r / 2}\right) \quad \text { as } n \rightarrow \infty .
\end{aligned}
$$


Theorem 4.2. Let $f(x)$ be as in the second paragraph of this section with the same notation, $U_{s}(y)$ and $V_{s}(y)$ being polynomials in $y$ of degree $u_{s}$ and $v_{s}$, respectively. Let $m_{-}$and $m_{+}$be the smallest integers for which

$$
r<\Re \alpha_{m_{-}} \text {and } r<\Re \beta_{m_{+}} .
$$

Then, with $h=(n+1 / 2)^{-2}$, it holds that

$$
\begin{aligned}
E_{n}[f]= & \sum_{s=0}^{m_{-}-1} \sum_{k=1}^{\left\lceil r / 2-\Re \alpha_{s}-1\right\rceil} U_{s}\left(D_{\alpha_{s}}\right)\left[c_{k}\left(\alpha_{s}\right) h^{\alpha_{s}+k}\right] \\
& +\sum_{s=0}^{m_{+}-1} \sum_{k=1}^{\left\lceil r / 2-\Re \beta_{s}-1\right\rceil} V_{s}\left(D_{\beta_{s}}\right)\left[c_{k}\left(\beta_{s}\right) h^{\beta_{s}+k}\right]+O\left(h^{r / 2}\right) \quad \text { as } n \rightarrow \infty .
\end{aligned}
$$

The proof of Theorem 4.1 is achieved precisely as that of Theorem 2.2 by modifying $p(x)$ in (3.1) as in

$$
p(x)=\sum_{s=0}^{m_{-}-1} A_{s}(1-x)^{\alpha_{s}}+\sum_{s=0}^{m_{+}-1} B_{s}(1+x)^{\beta_{s}} .
$$

Similarly, the proof of Theorem 4.2 is achieved precisely as that of Theorem 2.3 by modifying $p(x)$ in (3.19) as in

$$
p(x)=\sum_{s=0}^{m_{-}-1} U_{s}(\log (1-x))(1-x)^{\alpha_{s}}+\sum_{s=0}^{m_{+}-1} V_{s}(\log (1+x))(1+x)^{\beta_{s}} .
$$

In both cases, the functions $\phi(x):=f(x)-p(x)$ are in $C^{r}[-1,1]$ so that $E_{n}[\phi]=$ $O\left(h^{r / 2}\right)$ as $n \rightarrow \infty$. We leave the details to the reader.

Note that the summations over the $\alpha_{s}$ (the $\beta_{s}$ ) in (4.2) and (4.4) are empty in case $\Re \alpha_{0} \geq r / 2-1\left(\Re \beta_{0} \geq r / 2-1\right)$.

\section{REFERENCES}

[1] K.E. Atkinson. An Introduction to Numerical Analysis. Wiley, New York, 1978. MR504339 (80a:65001)

[2] P.J. Davis and P. Rabinowitz. Methods of Numerical Integration. Academic Press, New York, second edition, 1984. MR760629 (86d:65004)

[3] D. Elliott. Uniform asymptotic expansions of the Jacobi polynomials and an associated function. Math. Comp., 25:309-315, 1971. MR0294737(45:3805)

[4] I. Navot. An extension of the Euler-Maclaurin summation formula to functions with a branch singularity. J. Math. and Phys., 40:271-276, 1961. MR0140876 (25:4290)

[5] F.W.J. Olver. Asymptotics and Special Functions. Academic Press, New York, 1974. MR0435697 (55:8655)

[6] M.J.D. Powell. Approximation Theory and Methods. Cambridge University Press, Cambridge, 1981. MR604014 (82f:41001)

[7] A. Ralston and P. Rabinowitz. A First Course in Numerical Analysis. McGraw-Hill, New York, second edition, 1978. MR0494814 (58:13599)

[8] A. Sidi. Practical Extrapolation Methods: Theory and Applications. Number 10 in Cambridge Monographs on Applied and Computational Mathematics. Cambridge University Press, Cambridge, 2003. MR:1994507(2004e:65005)

[9] A. Sidi. Euler-Maclaurin expansions for integrals with endpoint singularities: a new perspective. Numer. Math., 98:371-387, 2004. MR2092747 (2005g:65012)

[10] J. Stoer and R. Bulirsch. Introduction to Numerical Analysis. Springer-Verlag, New York, third edition, 2002. MR 1923481 (2003d:65001)

[11] P. Verlinden. Acceleration of Gauss-Legendre quadrature for an integrand with an endpoint singularity. J. Comp. Appl. Math., 77:277-287, 1997. MR1440013 (98f:65029) 
Computer Science Department, Technion-Israel Institute of Technology, Haifa 32000, ISRAEL

E-mail address: asidi@cs.technion.ac.il

$U R L:$ http://www.cs.technion.ac.il/ ^asidi/ 